\title{
Rapid, specific, and sensitive detection of the ureR_1 gene in Klebsiella pneumoniae by loop-mediated isothermal amplification method
}

\author{
Chao Li (ii) ${ }^{1,2, *}$, Gongyu Fu (ii) ${ }^{1,2, *}$, Yaoqiang Shi (i) $^{1,2}$, A-Mei Zhang (i) $^{1,2}$, Xueshan Xia (ii) ${ }^{1,2}$,

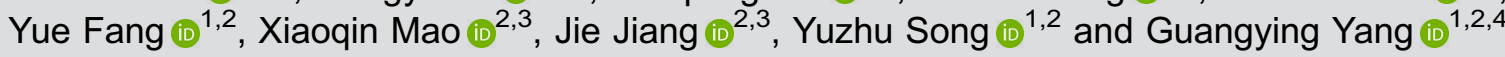 \\ ${ }^{1}$ Faculty of Life Science and Technology, Kunming University of Science and Technology, Kunming, Yunnan, China \\ ${ }^{2}$ Molecular Medicine Center of Yunnan Province, Kunming, Yunnan, China \\ ${ }^{3}$ Department of Clinical Laboratory, First People's Hospital of Yunnan Province, Kunming, Yunnan, China \\ ${ }^{4}$ Yunnan SciSpark Biotechnology Co. Ltd., Kunming, Yunnan, China
}

\begin{abstract}
Klebsiella pneumoniae is one of the main pathogenic bacteria that causes nosocomial infections, such as pneumonia, urinary tract infection, and sepsis. Therefore, the rapid and accurate detection of $K$. pneumoniae is important for the timely treatment of infectious patients. This study aimed to establish a loop-mediated isothermal amplification (LAMP) method for the rapid and sensitive detection of $K$. pneumoniae-specific gene ureR_1 (Gene ID: 11847803). The ureR_1 gene was obtained through local and online BLAST, and the specific primers were designed for its detection. Positive reactions were observed on all $140 \mathrm{~K}$. pneumoniae clinical isolates while all the 82 non-K. pneumoniae clinical isolates were negative. Plasmids with the specific gene and the mouse blood with $K$. pneumoniae were used for sensitivity analysis. The detection limit of the LAMP was 1 bacterium/ reaction. The results showed that the LAMP targeted to ure $R_{-} 1$ is a fast, specific, sensitive, inexpensive, and suitable method for the detection of K. pneumoniae.
\end{abstract}

Key words: Loop-mediated isothermal amplification; Polymerase chain reaction; Klebsiella pneumoniae; Novel specific gene; Specific; Sensitive method

\section{Introduction}

Klebsiella pneumoniae is a Gram-negative facultative anaerobic bacterium and an important conditional pathogen in hospitals over the past years (1-3). K. pneumoniae ubiquitously occurs in natural environments and is frequently found in the respiratory and gastrointestinal organs of patients $(4,5)$. Moreover, it is one of the main causes of nosocomial infections, which can lead to pneumonia, urinary tract infection, and sepsis (6). Children, older adults, hypoimmune individuals, and the patients undergoing longterm antibiotic therapy and intensive treatment are the susceptible population to K. pneumoniae. The World Health Organization reported an estimated 18.8 billion cases of pneumonia, along with upper respiratory tract infections, and about 4 million deaths in 2013 (7).

The prompt delivery of definitive therapy can prevent the spread of $K$. pneumoniae, control the inflammatory process, and decrease the mortality rate (8). Hence, the efficient and rapid detection of $K$. pneumoniae is deemed important. Conventional methods for the identification of K. pneumoniae in clinics include bacterial culture, immunological methods, and polymerase chain reaction (PCR) (9-11). Bacterial culture was considered the gold standard procedure for identifying $K$. pneumoniae $(12,13)$. After being infected by a pathogen, the specific antibody is formed in the host. Thus, immunological testing methods, including enzyme-linked immunosorbent assay, western blot, and immune chromatography, are based on the specificity of the antigen-antibody reaction. However, bacterial culture and immunological methods are time-consuming and exhibit low precision (14). Therefore, the PCR technique has been extensively used in clinical testing due to its high sensitivity and fast result (15). However, it is inconvenient in the clinical setting due to the requirement of isothermal cyclic amplification $(16,17)$. Although whole

Correspondence: Yuzhu Song: <yuzhusong@kmust.edu.cn> | Guangying Yang: <yguangying@foxmail.com>

${ }^{*}$ These authors contributed equally to this work.

Received September 21, 2018 | Accepted January 21, 2019 
genome sequencing has been recently identified as a precise method for studying pathogenic bacteria, the high costs of the necessary reagent and apparatus limit its universal application (18).

Loop-mediated isothermal amplification (LAMP) can be potentially used as a simple screening assay in the field by clinicians $(1,19)$. Compared with the other methods, LAMP technology has the advantages of simplicity, rapidity, and high sensitivity. Thus, it is more suitable for use in clinical laboratories. A novel specific gene of $K$. pneumoniae was obtained through bioinformatics analysis in this study (ureR_1) and an efficient and accurate LAMP method to detect it was built for the detection of $K$. pneumoniae, and validated by PCR assay.

\section{Material and Methods}

\section{Bacterial strains, culture conditions, and DNA extraction}

In this study, 140 K. pneumoniae clinical isolates and 82 non- $K$. pneumoniae strains were obtained from the First People's Hospital of Yunnan Province. The 82 nonK. pneumoniae strains included Escherichia coli $(n=15)$, Staphylococcus aureus $(\mathrm{n}=8)$, Staphylococcus epidermidis $(n=24)$, Enterococcus faecalis $(n=20)$, and Micrococcus luteus $(n=15)$. All strains were collected by the doctors from the Hospital and cultured in Luria-Bertani medium in an orbital shaker $\left(37^{\circ} \mathrm{C}\right.$, with $180 \mathrm{rpm}$, overnight). A bacterial genomic DNA kit (Zomanbio, China) and a direct-extraction reagent (20) were used to extract genomic DNA from bacteria. The extracted genomic DNA was stored at $-20^{\circ} \mathrm{C}$ for further use.

\section{Screening of $K$. pneumoniae specific genes}

First, the genomic sequence of $K$. pneumoniae subsp. pneumoniae HS11286 (GenBank No. NC_016845.1) and the known non-redundant nucleic acid database from National Center for Biotechnology Information (NCBI) were downloaded to the local server as the local database. Then, the potential specific genes of K. pneumoniae subsp. pneumoniae HS11286 were screened by sequence similarity alignment. Online BLAST was used to further identify the screened potential specific genes due to the slow update of the local database. Two-step strategies using interspecies-specific and intraspecies commonality were used to identify specific genes by online BLAST. The first one excluded $K$. pneumoniae during the alignment; the gene may be considered a possible target gene if the alignment result is different. The second included $K$. pneumoniae during alignment, and the highly conserved genes can be considered possible target genes. The retrieval range was limited to the species with known sequences except for $K$. pneumoniae. The specific gene of $K$. pneumoniae can only be considered when the alignment differs from those of other species, similar to those of a few species, or features a very low similarity.

\section{Primers design and reaction}

Four oligonucleotide primers (outer and inner primers, F3/B3 and FIP/BIP, respectively) targeting the specific gene were designed by the Primer Explorer V5 software (http: //primerexplorer.jp/lampv5/index.html) for LAMP assay. The outer primers (B3/F3) were also used in the PCR assays; the target fragment of the amplification was 203 base pairs (bp). Table 1 presents the primers used in this study. PCR was performed using $2 \times$ TSINGKE Master Mix, which was purchased from TSINGKE Biological Technology Company (China). According to the operating instructions, the PCR reaction system containing $12.5 \mu \mathrm{L}$ of $2 \times$ TSINGKE Master Mix, $1.0 \mu \mathrm{L}$ of primers $(10 \mu \mathrm{M})$, and $1.0 \mu \mathrm{g}$ of DNA template was added with nuclease-free water up to $25 \mu \mathrm{L}$ volume. The reactions were performed in a GeneAmp PCR System 9700 (Thermo Fisher Scientific, Inc., USA) with the following amplification conditions: predenaturation at $95^{\circ} \mathrm{C}$ for $5 \mathrm{~min}$, followed by 32 cycles, denaturation at $95^{\circ} \mathrm{C}$ for $30 \mathrm{~s}$, annealing at $57^{\circ} \mathrm{C}$ for $30 \mathrm{~s}$, extension at $72^{\circ} \mathrm{C}$ for $30 \mathrm{~s}$, and a final extension at $72^{\circ} \mathrm{C}$ for $7 \mathrm{~min}$. Five microliters of the PCR products were used in the $2 \%$ agarose gel electrophoresis at $120 \mathrm{~V}, 30 \mathrm{~min}$, and the agarose gel was stained by Gel stain (Beijing Transgen Biotech Co., Ltd., China). The LAMP reaction was carried out in a primer with a total volume of $25 \mu \mathrm{L}$. The system contained $12.5 \mu \mathrm{L} 2 \times$ Isothermal Master Mix (Great Britain), $8 \mu \mathrm{M}$ FIP and BIP, $1 \mu \mathrm{M}$ B3 and F3, $100 \mathrm{ng}$ genomic DNA, and up to $25 \mu \mathrm{L}$ nuclease-free water. The reaction was amplified using Genie ${ }^{B}$ II (OptiGene, UK) at

Table 1. Primers for the amplification of the ure $R_{-} 1$ gene.

\begin{tabular}{lcc}
\hline Primers & Sequence $\left(5^{\prime}-3^{\prime}\right)$ & Size $(\mathrm{bp})$ \\
\hline Outer primers & & \\
F3 & CCGATAGAGAACTCGAACTG & 20 \\
B3 & TCTGATGCATTTTACCCTGAT & 21 \\
Inner primers & & 49 \\
FIP & TCTTTGAAAAACCTTCGCTCCATATTTTTCTTCGCGCTAACTATCAACT & 48 \\
BIP & CATTCATATTGAAAAGCAGACCCGTTTTGCTCGATAAAGCCATGAGAA & 4 \\
\hline
\end{tabular}


$65^{\circ} \mathrm{C}$ for $30 \mathrm{~min}$, and then the primer was annealed at temperatures ranging from 80 to $89^{\circ} \mathrm{C}$. SYBR-Green I (Beijing Solarbio Science \& Technology Co., Ltd., China), a nuclear dye, was added into the LAMP reaction tubes for fluorescence visualization of the LAMP products.

\section{Construction of plasmids and preparation of blood template}

The positive plasmids were constructed by the following steps. The DNA fragment of ureR_1 was obtained by PCR, and the genome DNA of $K$. pneumoniae was used as a template. The ure $R \_1$ gene was then inserted into the pMD 19-T simple vector and transformed into JM109 competent cells. The positive clones were selected from the LB solid medium after overnight culture and used for plasmid extraction. Finally, the copies of recombinant plasmids were calculated by the deduced polynomial model described by the equation

$$
C=\frac{X \times 10^{-9}}{A+Y \times 324} \times N_{A}
$$

where $C$ denotes the copies of plasmids; $X$ and $Y$ represent the concentration of plasmids and the number of base pairs of the target fragment, respectively; $\mathrm{N}_{\mathrm{A}}$ is Avogadro's constant $\left(6.02 \times 10^{23}\right) ; A$ is the number of base pairs of the vector; and 324 represents the average molecular weight of each base pair.

To prepare the simulated samples from patients, $K$. pneumoniae suspension was mixed with whole blood from mice at a volume ratio of 1:1. The mixed blood sample was then lysed using a direct-extraction reagent for PCR and LAMP assays.

\section{Specificity of the PCR and LAMP reactions}

A total of 140 K. pneumoniae clinical isolates and 82 non-K. pneumoniae strains were used for the assessment of the PCR and LAMP reactions specificity. All genomic DNA of the tested strains were prepared by the TIANamp genomic DNA kit (Tiangen, China) for PCR and LAMP assays. The PCR test was used as the gold standard in preliminary experiments for the specificity test prior to the LAMP test. The LAMP test was used after validating the specificity by PCR.

\section{Sensitivity of the PCR and LAMP reactions}

In this study, the sensitivity of PCR and LAMP reactions were evaluated using two different templates, the serially diluted 10 -fold positive plasmids $\left(10^{9}-10^{0}\right.$ copies) and the blood sample $\left(10^{7}-10^{0}\right.$ bacteria) mimicking infection. The counted $K$. pneumoniae strain was serially diluted by 10 -fold and then mixed with mouse blood at $1: 1$ proportion to mimic infection. A heating method was used to extract DNA from the blood sample and the suspension was used as the template for PCR and LAMP assays.

\section{Results}

\section{Screening the specific gene}

Following the preliminary screening of possible specific genes by local BLAST, we obtained 700 potential specific genes. These genes were then used in the second screening by online BLAST. Conclusively, 4 potential specific genes were attained, and they met the criterion about the interspecific specificity and intraspecies universality. The primers for LAMP reaction were designed on the Primer Explorer V5 software (http: //primerexplorer.jp/ lampv5/index.html). In this step, four primers were designed for the six regions of the target gene in the LAMP, and the primers had to be confirmed by primer BLAST and PCR assay. With the above harsh selection conditions, the ureR_1 gene (GenBank ID: 11847803 ) was finally identified as the only one that can be used in the detection of $K$. pneumoniae. The ureR_1 gene was involved in the encoding of putative helix-turnhelix AraC-type transcriptional regulator (YP_005227085), and could be a new target for the identification of K. pneumoniae.

\section{Construction of the positive plasmid}

Positive plasmids with ureR_1 gene were constructed and verified by bacterial liquid PCR and sequencing. The positive clones were then cultured again for the extraction of positive plasmids. The plasmids were verified by agarose gel electrophoresis and stored at $-20^{\circ} \mathrm{C}$ for future use.

\section{Specificity of the PCR and LAMP reactions}

Prior to LAMP reaction, the PCR test was used for the specificity test. One hundred and forty $K$. pneumoniae strains and 82 non-K. pneumoniae strains were tested. All the non-K. pneumoniae were negative and all the K. pneumoniae strains were positive, the representative results are shown in Figure 1. After validation by PCR, LAMP was used to test the specificity. Figure 2 shows the amplification curve results of the LAMP, and Figure 3 shows the melt curve of the products in LAMP specificity reaction tubes. Figure 4 shows the fluorescence visualization of the LAMP reaction tubes, which were consistent with PCR results.

\section{Sensitivity of the PCR and LAMP reactions}

Figure 5 demonstrates the extreme sensitivities in PCR assay, which can reach up to $10^{\circ}$ copies per reaction. The LAMP results are shown in Figure 6. Figure 7 shows the melt curve of the products in LAMP sensitivity reaction tubes and Figure 8 shows the fluorescence visualization of the LAMP reaction tubes in the sensitivity test. According to Figures 5, 6, 7, and 8, both the PCR and LAMP assay exhibited a high sensitivity that can reach up to $10^{\circ}$ bacterium/reaction. 


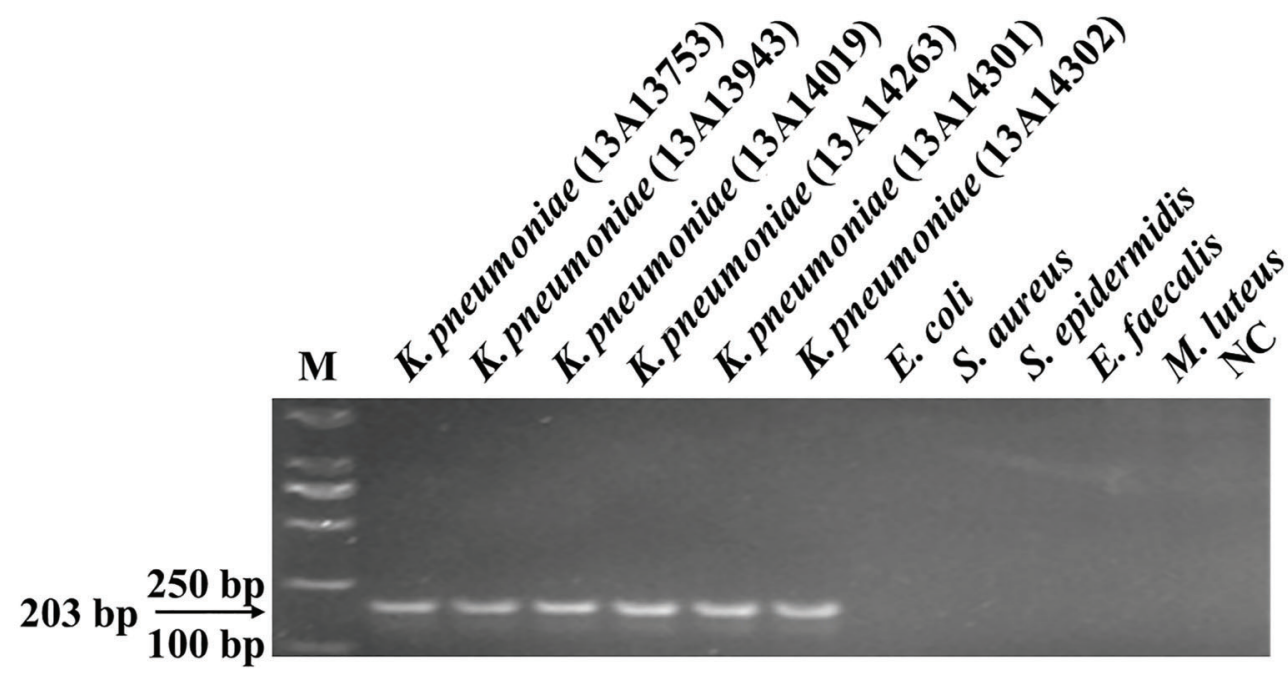

Figure 1. Specificity of the PCR assay for detecting the target gene ureR_1 using the primers B3/F3. Genomic DNA of $K$. pneumoniae was used as the template for PCR in lane 2 to lane 7 ; the template in lane 8 to lane 12 were ordinal of control strains, E. coli, S. aureus, S. epidermidis, E. faecalis, M. luteus. M: 2000 marker; NC: negative control, with sterile distilled water as the template. All experiments were repeated twice.

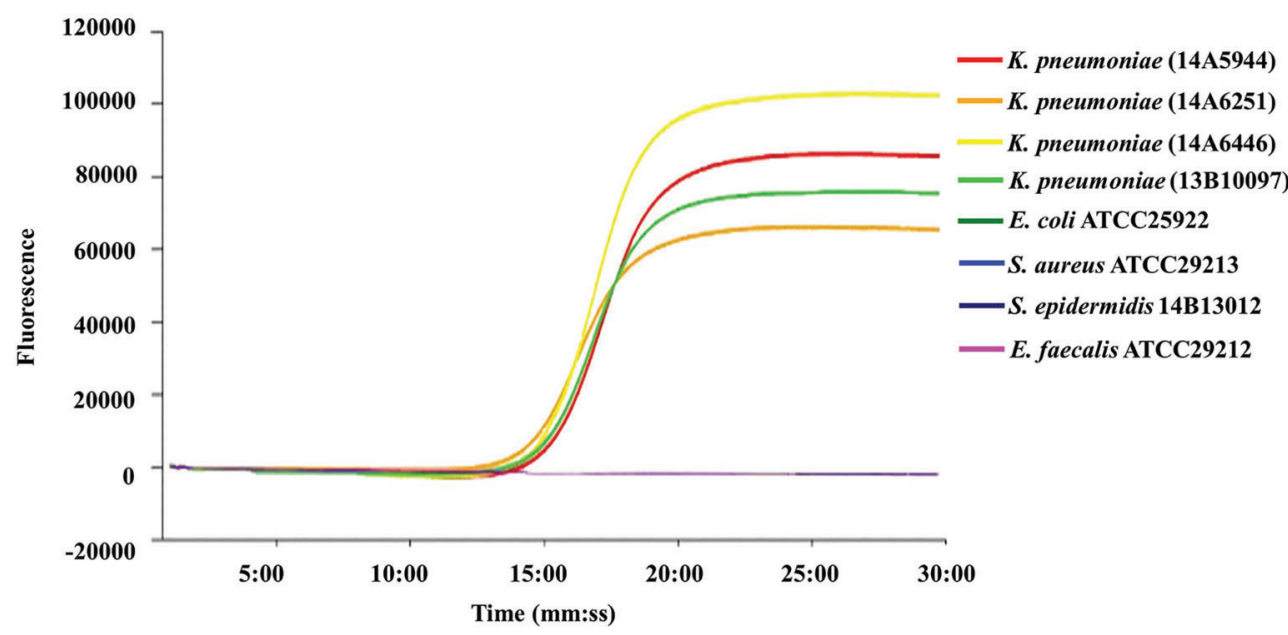

Figure 2. The amplification curve results of loop-mediated isothermal amplification (LAMP) specificity reaction. Specificity of the LAMP assay for detecting the target gene of ureR_1 by Genie ${ }^{\text {R }}$ II. Genomic DNA of K. pneumoniae was used as the template for the LAMP test. All experiments were repeated twice.

\section{Discussion}

K. pneumoniae is one of the main pathogenic bacteria that causes acute respiratory infections, which is the primary cause of child mortality in developing countries, accounting for approximately 3 million deaths annually (7). In China, K. pneumoniae was reported to account for $9.03 \%$ of total bacterial infection in hospitals (21-23). In the United States, Europe, and Africa, K. pneumoniae has become the main pathogenic bacterium (after Escherichia coli) in patients with pyogenic liver abscess over the past two decades. $K$. pneumoniae infection exhibits the tendency to spread worldwide and poses a serious threat to the lives of people $(22,24-26)$. Thus, early and accurate diagnosis can decrease the morbidity and mortality caused by $K$. pneumoniae infection, and a rapid and sensitive diagnostic method is urgently required.

Bacterial culture was the preferred method for identifying pathogenic bacterial infections in clinics. However, it was toilsome, slow, and poorly effective. Mass spectrometry 


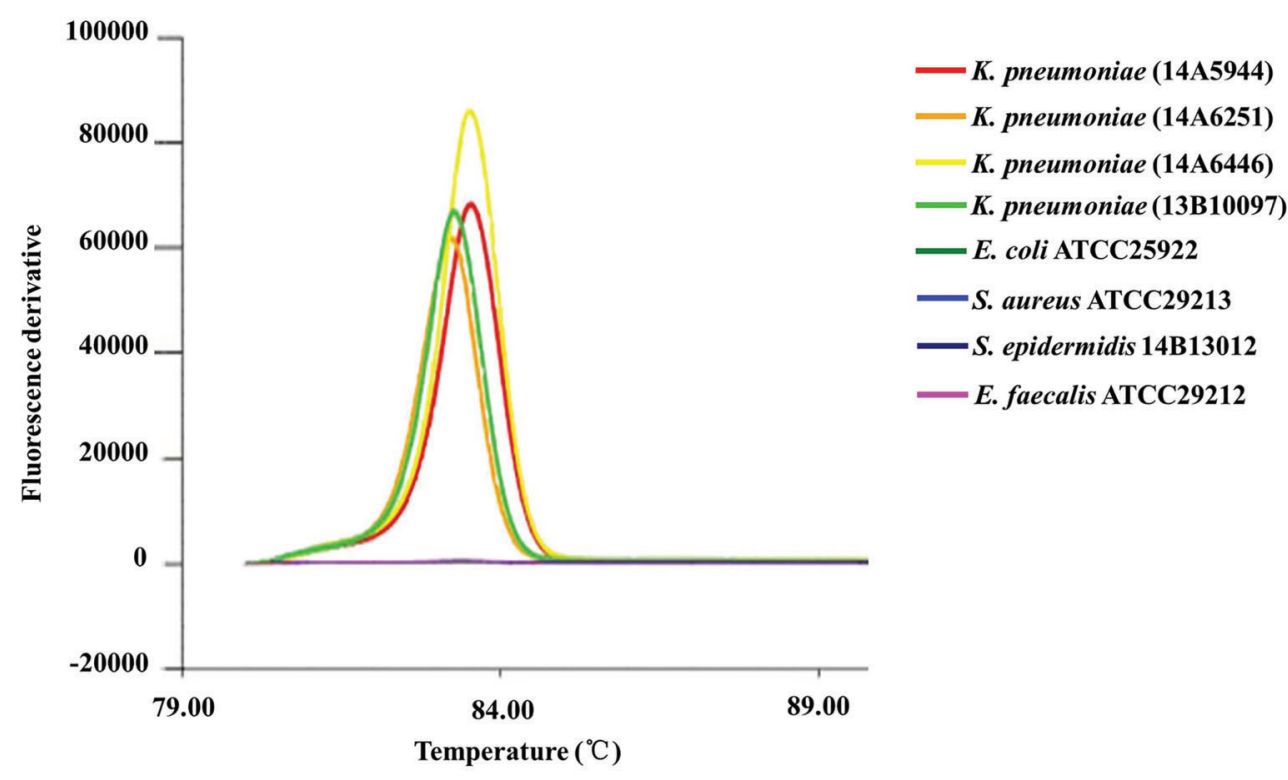

Figure 3. The melt curve in loop-mediated isothermal amplification (LAMP) specificity reaction tubes. Specificity of the LAMP assay for detecting the target gene of ureR_1 by Genie ${ }^{\mathbb{R}}$ II. Genomic DNA of K. pneumoniae was used as the template for LAMP test. All experiments were repeated twice.

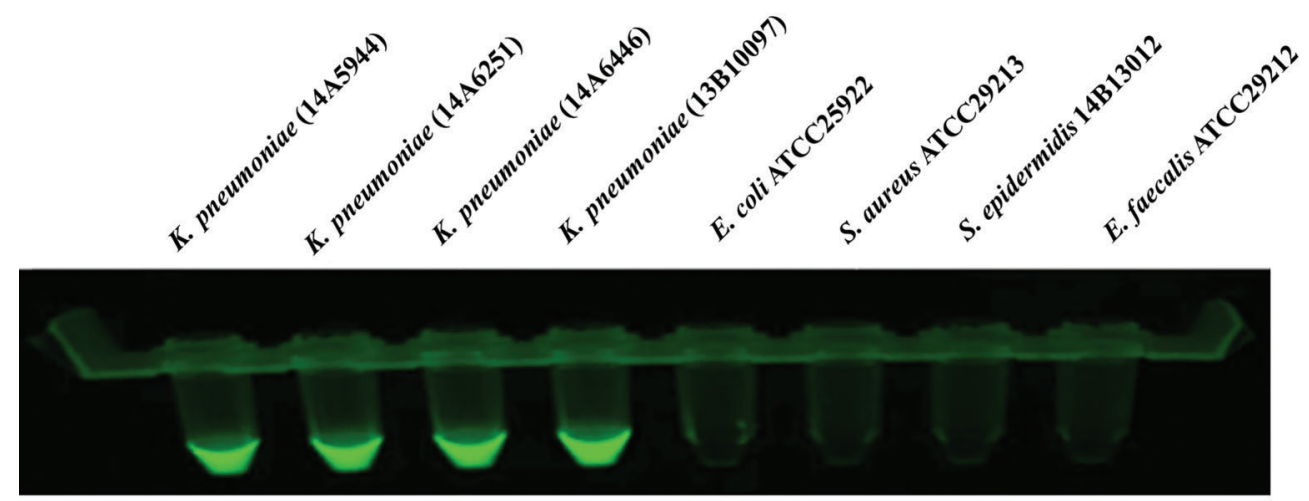

Figure 4. The fluorescence visualization of the loop-mediated isothermal amplification (LAMP) specificity reaction tubes. Specificity of the LAMP assay for detecting the target gene of ureR_1. Genomic DNA was used as the template for the LAMP. The results were observed under UV-light. All experiments were repeated twice.

is a high-speed approach used to detect pathogenic bacteria with higher accuracy than bacterial culture. However, mass spectrometry is expensive, and thus, it is not suitable for all types of hospitals. Numerous methods have been used to detect $K$. pneumoniae in clinics. PCR-based methods are widely used to detect $K$. pneumoniae by amplifying specific genes, including $r c s A$, tyrB, gapA, and 16S rRNA ITS (27$30)$. However, a high-precision thermal cycler is needed, which cannot be afforded by low-level medical institutions. By contrast, LAMP, which exhibits fast and accurate characteristics, has been widely used in clinical laboratories to detect pathogens, including bacteria, viruses, fungi, and parasites (31-33), although this technology is relatively new.
In the present work, a conservative specific gene was identified to detect $K$. pneumoniae. The results in Figure 1 suggest that the ure $R \_1$ gene was specific to $K$. pneumoniae and can be used as a biomarker in clinics. In many studies, the sensitivity of LAMP was reported to be 10-fold higher than that of the PCR method $(27,29,34)$. However, specificity and sensitivity between LAMP and PCR were similar when the ureR_1 gene was used. These results suggest that the ure $R \_1$ gene and the primers designed in this study may be more specific and sensitive than those in other studies $(27,35)$. Also, only 32 min was needed for the identification of $K$. pneumoniae by the LAMP method, while 

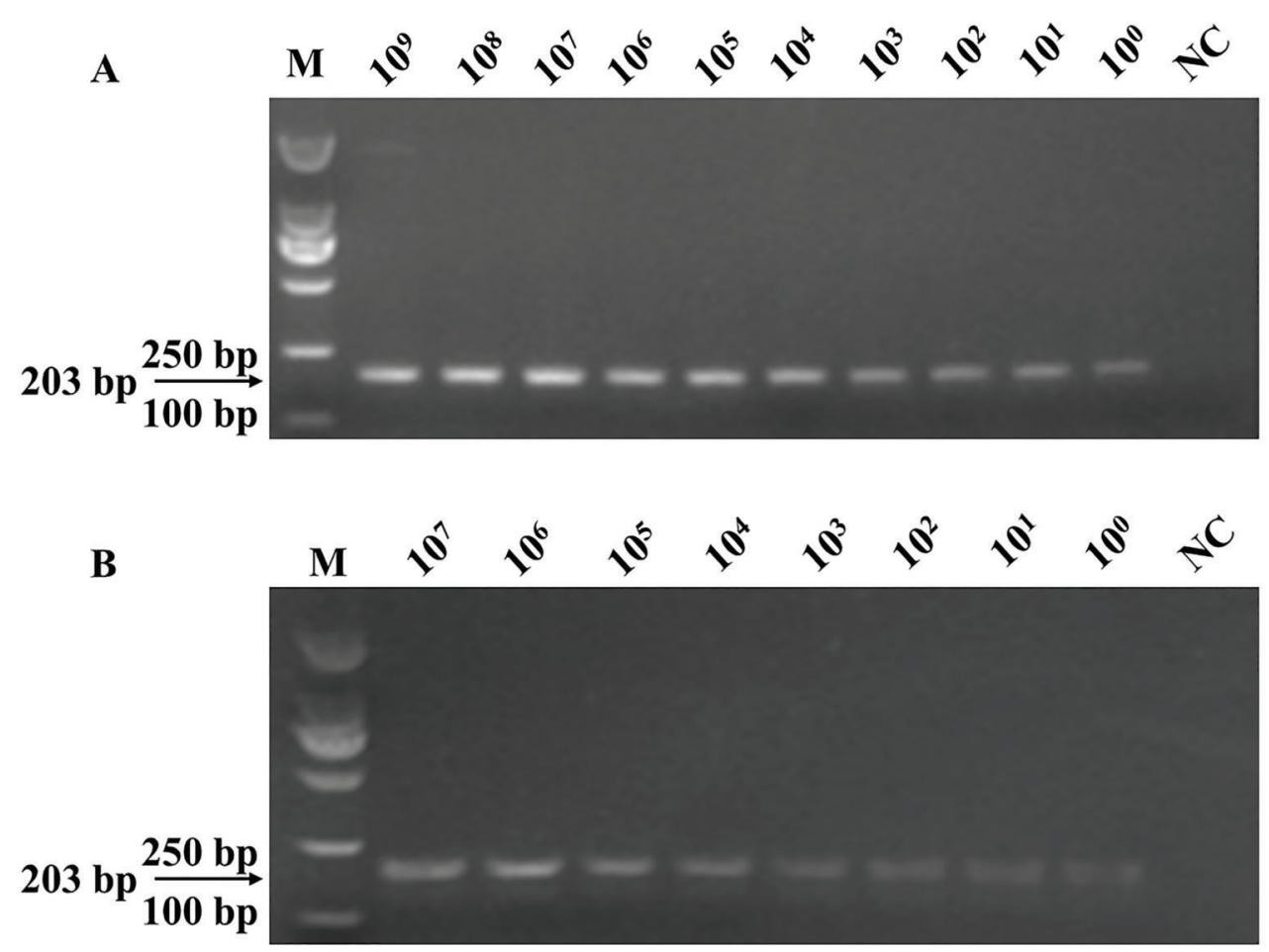

Figure 5. A, Sensitivity of the PCR assay for detecting the target gene of ureR_1 using the primers FIP/BIP. The positive plasmids of $K$. pneumoniae were serially diluted 10-fold as templates for PCR assay. B, Bacterial solutions were serially diluted 10-fold with the mouse blood with a volume ratio of 1:1. These mixtures were lysed and then the suspension was used for PCR assay. M: 2000 marker; NC: negative control. All experiments were repeated twice.

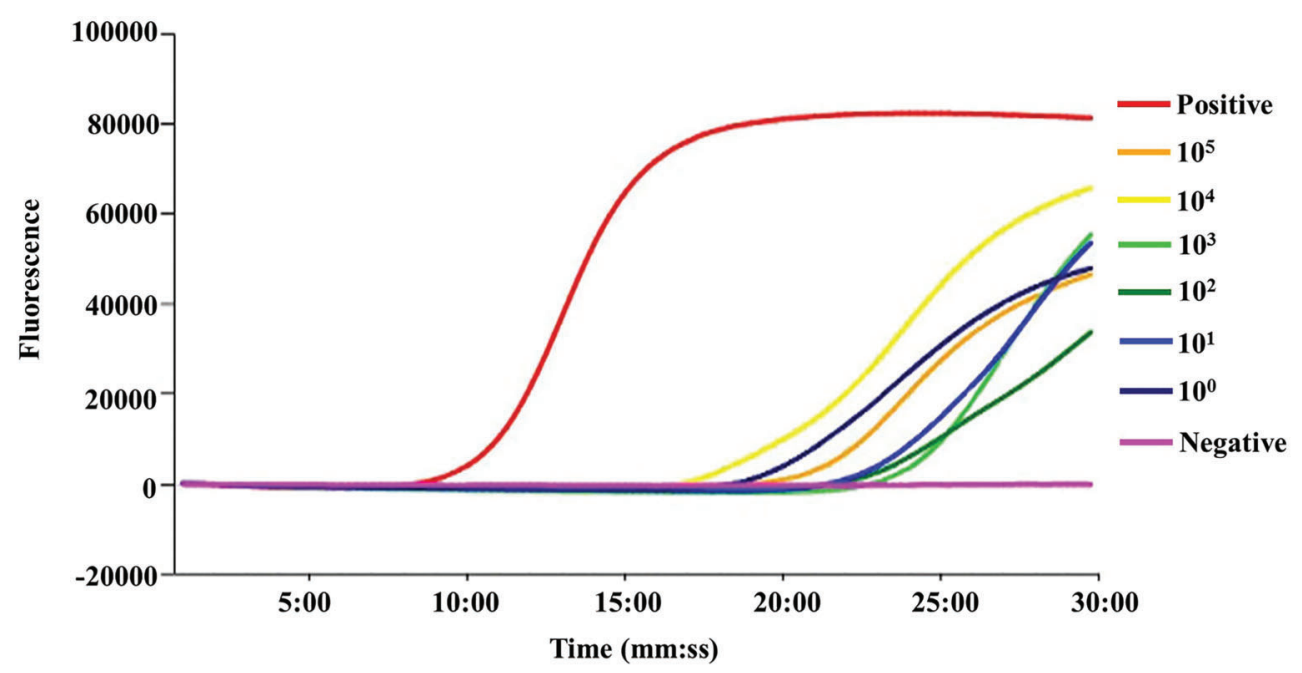

Figure 6. Sensitivity of the loop-mediated isothermal amplification (LAMP) reactions. The bacterial solutions were serially diluted 10-fold with the mouse blood with a volume ratio of 1:1. These mixtures were lysed and then the suspension was used for the LAMP assay. The positive plasmid was the positive control and water was the negative control. The concentration of bacterium was from $10^{5}-10^{0}$. All the experiments were repeated twice. 


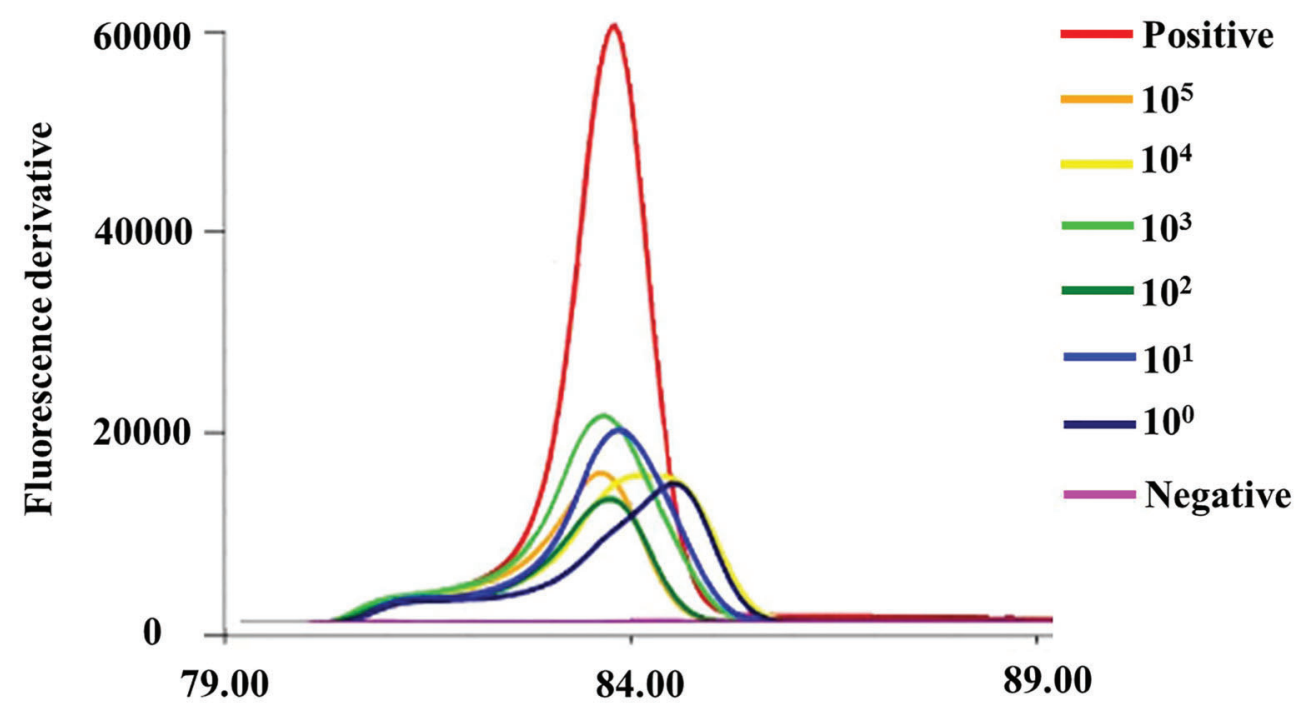

Temperature $\left({ }^{\circ} \mathrm{C}\right)$

Figure 7. The melt curve of the products in loop-mediated isothermal amplification (LAMP) sensitivity reaction tubes for detecting the target gene of ureR_1 by Genie ${ }^{\mathbb{R}}$ II. The lysed suspension of $K$. pneumoniae and mouse blood were used as the template for LAMP test. The positive plasmid was the positive control and water was the negative control. The concentration of bacterium was from $10^{5}-10^{0}$. All experiments were repeated twice.

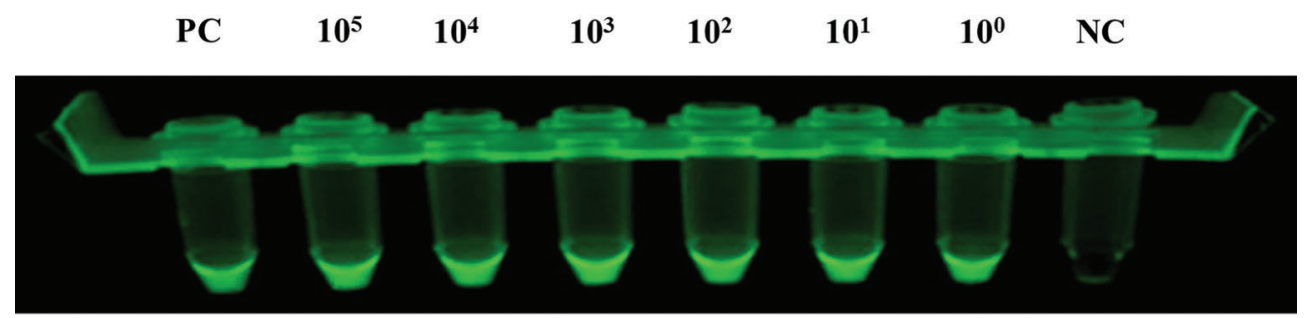

Figure 8. Fluorescence visualization of the loop-mediated isothermal amplification (LAMP) reaction tubes in the sensitivity test for detecting the target gene of ureR_1. The lysed suspension of $K$. pneumoniae and mouse blood was used as the template for the LAMP test. The positive plasmid was the positive control (PC) and water was the negative control (NC). The concentration of bacterium was from $10^{5}-10^{\circ}$. The results were observed under UV-light. All experiments were repeated twice.

90 min are required in PCR. In addition, the LAMP reaction results can be easily observed under UV-light. The LAMP method can greatly reduce the time required for identification.

In conclusion, we identified ure $R_{-} 1$ as a specific gene of $K$. pneumoniae and established a rapid, specific, and sensitive LAMP method using ureR_1 primers for the detection of K. pneumoniae. The established method may be extensively used in clinics in the future due to its

\section{References}

1. Lai YC, Peng HL, Chang HY. RmpA2, an activator of capsule biosynthesis in Klebsiella pneumoniae CG43, regulates K2 high specificity and sensitivity, easy visualization, and rapid results.

\section{Acknowledgments}

This work was supported by grants from Yunnan Science and Technology Commission (2015BC001 and 2015DH010). cps gene expression at the transcriptional level. $J$ Bacteriol 2003; 185: 788-800, doi: 10.1128/JB.185.3.788-800.2003. 
2. Yang XJ, Wang S, Cao JM, Hou JH. Draft genome sequence of klebsiella pneumoniae strain as isolated from Zhejiang Provincial Hospital of TCM, China. Genome Announc 2016; 4: pii: e00930-16, doi: 10.1128/genomeA.00930-16.

3. Kumar V, Park S. Potential and limitations of Klebsiella pneumoniae as a microbial cell factory utilizing glycerol as the carbon source. Biotechnol Adv 2018; 36: 150-167, doi: 10.1016/j.biotechadv.2017.10.004.

4. Podschun R, Ullmann U. Klebsiella spp. as nosocomial pathogens: epidemiology, taxonomy, typing methods, and pathogenicity factors. Clin Microbiol Rev 1998; 11: 589-603, doi: 10.1128/CMR.11.4.589.

5. Bednarz-Misa I, Serek P, Dudek B, Pawlak A, BuglaPloskonska G, Gamian A. Application of zwitterionic detergent to the solubilization of Klebsiella pneumoniae outer membrane proteins for two-dimensional gel electrophoresis. J Microbiol Methods 2014; 107: 74-79, doi: 10.1016/j.mimet. 2014.09.004.

6. Rock C, Thom KA, Masnick M, Johnson JK, Harris AD, Morgan DJ. Frequency of Klebsiella pneumoniae carbapenemase (KPC)-producing and non-KPC-producing Klebsiella species contamination of healthcare workers and the environment. Infect Control Hosp Epidemiol 2014; 35: 426-429, doi: 10.1086/675598.

7. Ferkol $\mathrm{T}$, Schraufnagel $\mathrm{D}$. The global burden of respiratory disease. Ann Am Thoracic Soc 2014; 11: 404-406, doi: 10. 1513/AnnalsATS.201311-405PS.

8. Wang F, Li R, Shang Y, Wang C, Wang GQ, Zhou DX, et al. A pilot study of quantitative loop-mediated isothermal amplification-guided target therapies for hospital-acquired pneumonia. Chin Med J 2016; 129: 181-186, doi: 10.4103/ 0366-6999.173484.

9. Bachman MA, Breen P, Deornellas V, Mu Q, Zhao L, Wu W, et al. Genome-wide identification of Klebsiella pneumoniae fitness genes during lung infection. mBio 2015; 6: e00775, doi: $10.1128 / \mathrm{mBio} .00775-15$.

10. Pongsachareonnont $P$, Honglertnapakul W, Chatsuwan T. Comparison of methods for identifying causative bacterial microorganisms in presumed acute endophthalmitis: conventional culture, blood culture, and PCR. BMC Infect Dis 2017; 17: 165, doi: 10.1186/s12879-017-2264-5.

11. Ahn JG, Choi SY, Kim DS, Kim KH. Enhanced detection and serotyping of Streptococcus pneumoniae using multiplex polymerase chain reaction. Korean J Pediatr 2012; 55: 424-429, doi: 10.3345/kjp.2012.55.11.424.

12. Hudu SA, Alshrari AS, Syahida A, Sekawi Z. Cell culture, technology: enhancing the culture of diagnosing human diseases. J Clin Diagn Res 2016; 10: DE01-5, doi: 10.7860/ JCDR/2016/15837.7460.

13. Kloss S, Kampe B, Sachse S, Rosch P, Straube E, Pfister $W$, et al. Culture independent Raman spectroscopic identification of urinary tract infection pathogens: a proof of principle study. Anal Chem 2013; 85: 9610-9616, doi: 10.1021/ac401 $806 f$.

14. Nakano R, Nakano A, Ishii Y, Ubagai T, Kikuchi-Ueda T, Kikuchi $\mathrm{H}$, et al. Rapid detection of the Klebsiella pneumoniae carbapenemase (KPC) gene by loop-mediated isothermal amplification (LAMP). J Infect Chemother 2015; 21: 202-206, doi: 10.1016/j.jiac.2014.11.010.

15. Abdoli A, Dalimi A, Soltanghoraee H, Ghaffarifar F. Molecular detection of Toxoplasma gondii in house sparrow
(Passer domesticus) by LAMP and PCR methods in Tehran, Iran. J Parasit Dis 2016; 40: 1317-1321, doi: 10.1007/s12639015-0680-2.

16. Ali ME, Razzak MA, Hamid SB, Rahman MM, Amin MA, Rashid NR, et al. Multiplex PCR assay for the detection of five meat species forbidden in Islamic foods. Food Chem 2015; 177: 214-224, doi: 10.1016/j.foodchem.2014.12.098.

17. Bharathi MJ, Murugan N, Rameshkumar G, Ramakrishnan $\mathrm{R}$, Venugopal Reddy YC, Shivkumar C, et al. Comparative evaluation of uniplex, nested, semi-nested, multiplex and nested multiplex PCR methods in the identification of microbial etiology of clinically suspected infectious endophthalmitis. Curr Eye Res 2013; 38: 550-562, doi: 10.3109/02713 683.2013.772205.

18. Long SW, Williams D, Valson C, Cantu CC, Cernoch P, Musser JM, et al. A genomic day in the life of a clinical microbiology laboratory. J Clin Microbiol 2013; 51: 12721277, doi: 10.1128/JCM.03237-12.

19. Notomi $\mathrm{T}$, Okayama $\mathrm{H}$, Masubuchi $\mathrm{H}$, Yonekawa $\mathrm{T}$, Watanabe $\mathrm{K}$, Amino $\mathrm{N}$, et al. Loop-mediated isothermal amplification of DNA. Nucleic Acids Res 2000; 28: E63, doi: 10.1093/nar/28. 12.e63.

20. Brewster JD, Paoli GC. DNA extraction protocol for rapid PCR detection of pathogenic bacteria. Anal Biochem 2013; 442: 107-109, doi: 10.1016/j.ab.2013.07.013.

21. Miriagou V, Cornaglia G, Edelstein M, Galani I, Giske CG, Gniadkowski M, et al. Acquired carbapenemases in Gramnegative bacterial pathogens: detection and surveillance issues. Clin Microbiol Infect 2010; 16: 112-122, doi: 10.1111/ j.1469-0691.2009.03116.x.

22. Liu Y, Wang JY, Jiang W. An increasing prominent disease of klebsiella pneumoniae liver abscess: etiology, diagnosis, and treatment. Gastroenterol Res Pract 2013; 2013: 258 514, doi: 10.1155/2013/258514.

23. Basu S. Klebsiella pneumoniae: An emerging pathogen of pyogenic liver abscess. Oman Med J 2009; 24: 131-133, doi: 10.5001/omj.2009.28.

24. Yonetani S, Ohnishi H, Ohkusu K, Matsumoto T, Watanabe T. Direct identification of microorganisms from positive blood cultures by MALDI-TOF MS using an in-house saponin method. Int J Infect Dis 2016; 52: 37-42, doi: 10.1016/j. ijid.2016.09.014.

25. Hou Y, Zhang X, Hou X, Wu R, Wang Y, He X, et al. Rapid pathogen identification using a novel microarray-based assay with purulent meningitis in cerebrospinal fluid. Sci Rep 2018; 8: 15965, doi: 10.1038/s41598-018-34051-0.

26. Alfaresi M, Mahboub $B$. Identification of bacteria in the sputum of a cystic fibrosis patient; a comparison of phenotypic and molecular methods. Open Microbiol J 2017; 11: 384-386, doi: 10.2174/1874285801711010384.

27. Dong D, Liu W, Li H, Wang Y, Li X, Zou D, et al. Survey and rapid detection of Klebsiella pneumoniae in clinical samples targeting the rcsA gene in Beijing, China. Front Microbiol 2015; 6: 519, doi: 10.3389/fmicb.2015.00519.

28. Hirama T, Minezaki S, Yamaguchi T, Kishi E, Kodama K, Egashira $\mathrm{H}$, et al. HIRA-TAN: a real-time PCR-based system for the rapid identification of causative agents in pneumonia. Respir Med 2014; 108: 395-404, doi: 10.1016/j.rmed.2013. 11.018.

29. Jeong ES, Lee KS, Heo SH, Seo JH, Choi YK. Rapid identification of Klebsiella pneumoniae, Corynebacterium 
kutscheri, and Streptococcus pneumoniae using triplex polymerase chain reaction in rodents. Exp Anim 2013; 62: 35-40, doi: 10.1538/expanim.62.35.

30. Tocchioni F, Tani C, Bartolini L, Moriondo M, Nieddu F, Pecile $P$, et al. The role of dna amplification and cultural growth in complicated acute appendicitis. Pediatr Rep 2016; 8: 6487, doi: 10.4081/pr.2016.6487.

31. Ohtsuka K, Yanagawa K, Takatori K, Hara-Kudo Y. Detection of Salmonella enterica in naturally contaminated liquid eggs by loop-mediated isothermal amplification, and characterization of Salmonella isolates. Appl Environ Microbiol 2005; 71: 6730-6735, doi: 10.1128/AEM.71.11.6730-6735.2005.

32. Ptaszynska AA, Borsuk G, Wozniakowski G, Gnat S, Malek W. Loop-mediated isothermal amplification (LAMP) assays for rapid detection and differentiation of Nosema apis and
N. ceranae in honeybees. FEMS Microbiol Lett 2014; 357: 40-48, doi: 10.1111/1574-6968.12521.

33. Soliman H, Saleh M, El-Matbouli M. Detection of fish pathogens by loop-mediated isothermal amplification (LAMP) technique. Methods Mol Biol 2015; 1247: 163-173, doi: 10. 1007/978-1-4939-2004-4.

34. Kitamura M, Aragane M, Nakamura K, Watanabe K, Sasaki Y. Rapid identification of drug-type strains in Cannabis sativa using loop-mediated isothermal amplification assay. J Nat Med 2017; 71: 86-95, doi: 10.1007/s11418-016-1031-z.

35. Kinoshita $\mathrm{Y}$, Niwa $\mathrm{H}$, Katayama $\mathrm{Y}$. Use of loop-mediated isothermal amplification to detect six groups of pathogens causing secondary lower respiratory bacterial infections in horses. Microbiol Immunol 2015; 59: 365-370, doi: 10.1111/ 1348-0421.12257. 